\title{
CENTRAL BLACK HOLES AND DARK HALOS IN ELLIPTICAL GALAXIES
}

\author{
P.T. DE ZEEUW \\ Sterrewacht Leiden \\ Postbus 9513, 2300 RA Leiden, The Netherlands \\ (tim@strw.LeidenUniv.nl)
}

Studies of the mass-to-light ratio $\mathrm{M} / \mathrm{L}$ as a function of radius in a galaxy usually focus on: a) the existence of dark matter in and around galaxies, and b) the presence of massive black holes in their nuclei. Here we concentrate on elliptical galaxies, and summarize some recent work in both areas. We refer to more extensive reviews for earlier developments (Schwarzschild, 1954; Trimble, 1987; Ashman, 1992; Kormendy, 1993). A third area of interest is the behavior of the stellar populations as a function of radius, and its effect on M/L (e.g., Peletier, 1989). We will not discuss this here.

\section{Central Black Holes}

Most models of the energy production in quasars and active galactic nuclei invoke the presence of a nuclear black hole with mass $M_{\mathrm{BH}}$ in the range $10^{6}$ to $10^{9} M_{\odot}$. The number of quasars at high redshift indicates that many normal galaxies we observe today must have gone through an active phase-if the lifetime of activity is short. Massive black holes could hence be common in 'normal' galactic nuclei (Lynden-Bell, 1969; Rees, 1984).

A massive central black hole significantly influences the motion of the surrounding stars out to a radius $r_{\mathrm{BH}}=G M_{\mathrm{BH}} / \sigma^{2}$, where $\sigma$ is a characteristic velocity dispersion of the stars in the center of the host galaxy. The density of stars inside $r_{\mathrm{BH}}$ has a power-law cusp, i.e., $\rho(r) \propto r^{-\alpha}$ with $\alpha$ between $3 / 2$ and $9 / 4$, depending on the details of the black hole formation scenario (Bahcall and Wolff, 1976; Young, 1980; Quinlan et al., 1994). The rms velocity $\left\langle v^{2}\right\rangle^{1 / 2}$ and the circular velocity $v_{c}$ diverge proportional to $r^{-1 / 2}$ when $r \rightarrow 0$. Typical values of $M_{\mathrm{BH}}$ and $\sigma$ lead to $r_{\mathrm{BH}} \lesssim 1^{\prime \prime}$ in nearby galaxies. As a result, high resolution photometric and kinematic ob- 
servations are required to find direct dynamical evidence for the existence of massive central black holes.

Surface brightness cusps have been observed in many elliptical galaxies. Ground-based measurements (Lauer, 1985; Kormendy, 1987) have been superseded by HST data (Lauer et al, 1991, 1992a, b; Stiavelli et al., 1993; Crane et al., 1993; Ferrarese et al., 1994; Kormendy et al., 1994). These cusps are consistent with the presence of central black holes but do not prove their existence, since other processes also give rise to very high central densities (e.g., Kormendy, 1993).

Ground-based spectroscopic observations have provided tantalizing but inconclusive hints for a central dark mass in the nuclei of half-a-dozen nearby galaxies. The evidence rests on an inwards increase of the lineof-sight velocity dispersion $\sigma_{\text {los }}$, and on a steep central gradient in the mean line-of-sight velocity $\left\langle v_{\text {los }}\right\rangle$. Such a $\left\langle v_{\text {los }}\right\rangle$-gradient is expected near a massive black hole, but it can also be the signature of a rapidly rotating nuclear stellar disk, of the kind seen in some nearby galaxies (van den Bosch et al., 1994; Kormendy et al., 1994). The claimed masses of the putative central black holes span several decades, and are correlated tightly with the distance to the host galaxy. The ground-based kinematic measurements invariably differ by at most $30 \%$ from constant M/L models (Rix, 1993).

Most estimates of black hole masses are based on spherical or isotropic dynamical models, while the host galaxies are usually flattened by $20 \%$ or more. The dynamics of spherical galaxies differs markedly from that of axisymmetric systems, let alone triaxial ones. High spatial and spectral resolution observations reveal line-of-sight velocity distributions with an excess of stars at low $v_{\text {los }}$ (Bender, 1990; van der Marel and Franx, 1993; van der Marel et al., 1994a; Bender et al., 1994). The standard Gauss fits to these velocity profiles (VPs) hence provide incorrect estimates of $\left\langle v_{\text {los }}\right\rangle$ and $\sigma_{\text {los }}$. Furthermore, the VPs are not the result of projection and seeing convolution of locally Gaussian velocity distributions. Comparing Gaussian VP fits to solutions of the spherical Jeans equation can easily introduce errors of $20-30 \%$, and the results should be treated with caution.

The compact E3 galaxy M32 has been scrutinized carefully for the presence of a nuclear black hole (Tonry, 1987; Richstone et al., 1990; Lauer et $a l ., 1992 \mathrm{~b})$. It shows no sign of nuclear activity or emission-line gas, and has no isophote twist, ellipticity variation, or color gradient in the inner $10^{\prime \prime}(35 \mathrm{pc})$. The VPs derived from the high resolution ground-based spectroscopy of van der Marel et al. (1994a) have recently been compared to the predictions of flattened axisymmetric dynamical models with distribution functions of the form $f\left(E, L_{z}\right)$, where $E$ is the orbital energy and $L_{z}$ is the orbital angular momentum component parallel to the rotation axis (van der Marel et al., 1994b; Qian et al., 1994; Dehnen, 1994). Without a black 

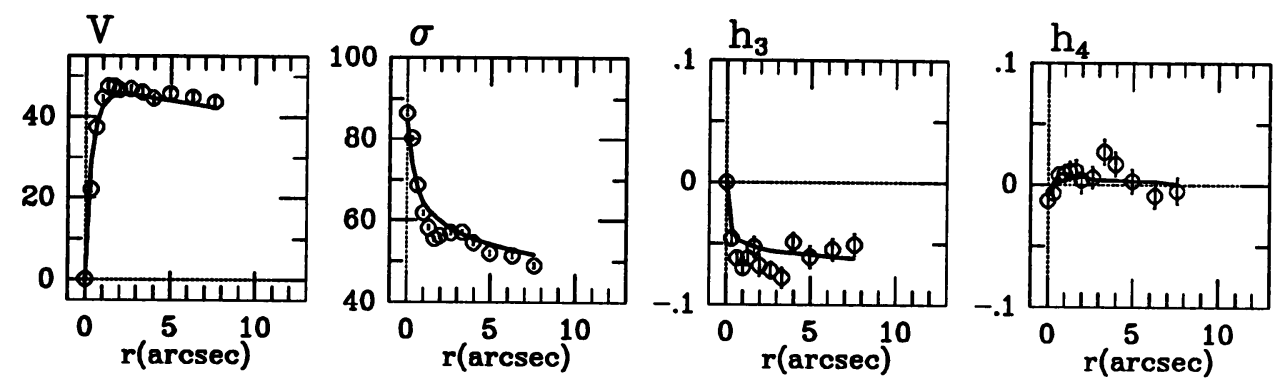

Figure 1. The data points are the observed VP parameters as a function of projected radius $r$ in arcsec along the major axis of M32 (van der Marel et al., 1994a). From left to right: the mean $V$ and dispersion $\sigma$ of the best fitting Gaussian to the VP, and the Gauss-Hermite moments $h_{3}$, which measures the lowest order antisymmetric deviations from a Gaussian, and $h_{4}$, which measures the lowest order symmetric deviations. The solid curves are the prediction of a two-integral $f\left(E, L_{z}\right)$ axisymmetric model with a $1.8 \times 10^{6} M_{\odot}$ central black hole, taking into account the seeing convolution and spatial binning of the observations. Equally good fits are obtained along the other four position angles where data of similar quality is available (Qian et al., 1994).

hole, no such two-integral model can fit the observed central peak in $\sigma_{\text {los }}$, nor the steep central $\left\langle v_{\text {los }}\right\rangle$ gradient. However, an $f\left(E, L_{z}\right)$ model with a central black hole of $1.8 \times 10^{6} M_{\odot}$ provides an astoundingly good fit to all available photometric and kinematic measurements (Fig. 1). The success of the $f\left(E, L_{z}\right)$-model does not prove that M32 has a large central dark mass. It remains to be seen whether a three-integral model without a black hole can be constructed, or ruled out. The measured VPs show already that a model without a central dark mass must combine a tangentially anisotropic velocity distribution outside $3 \mathrm{pc}$ with a strongly radially anisotropic velocity distribution inside $3 \mathrm{pc}$. Even if such a model can be constructed with a distribution function $f \geq 0$, it is not clear whether it will be stable, or even plausible. HST/FOS observations will allow the case for a dark mass to be strengthened further (Qian et al., 1994).

Until recently, the dynamical case for a supermassive black hole was weakest in M87, a well-known active E0 galaxy. The stellar $\sigma_{\text {los }}$ increases from $270 \mathrm{~km} / \mathrm{s}$ at $15^{\prime \prime}$ to $400 \mathrm{~km} / \mathrm{s}$ at $0.5^{\prime \prime}$ from the nucleus (van der Marel, 1994). Radially anisotropic spherical dynamical models fit the data without a black hole, while less anisotropic models with a central black hole of mass $\lesssim 5 \times 10^{9} M_{\odot}$ also fit the data, in agreement with earlier studies based on lower resolution data. The emission-line gas in the nucleus of M87 shows evidence for bulk motion, and for a strong inwards increase of the gas velocity dispersion: from $125 \mathrm{~km} / \mathrm{s}$ at $2^{\prime \prime}$ from the center to over $500 \mathrm{~km} / \mathrm{s}$ at $0.6^{\prime \prime}$. This is consistent with $M_{\mathrm{BH}} \sim 3 \times 10^{9} M_{\odot}$ (van der Marel, 1994). HST measurements show that this gas resides in a small nuclear disk. FOS 
spectra taken in two $0.26^{\prime \prime}$ apertures centered at $0.25^{\prime \prime}$ on each side of the nucleus indicate $v_{c} \sim 550 \mathrm{~km} / \mathrm{s}$ (Harms et al., 1994). This makes the case for a $\sim 3 \times 10^{9} M_{\odot}$ black hole in M87 very compelling.

\section{Dark Halos}

The existence of the fundamental plane (Djorgovski and Davis, 1987) implies that the global $\mathrm{M} / \mathrm{L}$ of elliptical galaxies varies systematically with total luminosity $\mathcal{L}$ and effective radius $R_{e}$ (Dressler et al., 1987). If the observed $\mathrm{M} / \mathrm{L}$ values are caused by a normal stellar population, then this variation may be set by the physics of star formation. It is also possible that dark matter contributes significantly to $M / L$ in the luminous regions. In this case the fundamental plane must result from a "conspiracy" between the luminous and dark material in order to produce such similar M/L 's for a large variety of systems. The dark matter content of ellipticals therefore can shed light on their formation.

\subsection{ABSORPTION LINE MEASUREMENTS}

A number of studies have sought to use the behavior of $\sigma_{\text {los }}$ with radius to detect unseen mass at large radii. Measurements of $\sigma_{\text {los }}$ beyond one $R_{e}$ are available for about a dozen elliptical galaxies, most of them nearly round objects (Efstathiou et al., 1982; Carollo, 1993; Saglia et al., 1993; Bertin et $a l ., 1994)$. The $\sigma_{\text {los }}$ profiles are flat in some cases, and spherical modeling based on distribution functions suggests either an outwards increase of M/L , or a constant $\mathrm{M} / \mathrm{L}$ at a value consistent with normal stellar populations and an increasingly tangentially anisotropic velocity distribution. The $\sigma_{\mathrm{los}}-$ profiles fall gently in other cases, and are consistent with constant M/L and at most a mild velocity anisotropy. Many E0 galaxies show evidence for rotation: $\left\langle v_{\text {los }}\right\rangle / \sigma_{\text {los }}$ steadily increases outwards. This can be included in the $\mathrm{M} / \mathrm{L}$ determination by modeling $\left\langle v_{\text {los }}^{2}\right\rangle=\sigma_{\text {los }}^{2}+\left\langle v_{\text {los }}\right\rangle^{2}$, but it suggests that these systems are triaxial (Levison, 1987). The results of spherical modeling therefore are suspect.

This unsatisfactory situation is improving now that the entire velocity profile VP can be measured at radii beyond one $R_{e}$. This constrains the anisotropy (Dejonghe, 1987; Gerhard, 1991, 1993), and provides tighter bounds on M/L . Carollo (1993) has measured VP shapes of four elliptical galaxies out to $\gtrsim 2 R_{e}$. Fig. 2 shows the result for the non-rotating E0 galaxy NGC 2434 . The dispersion profile is flat beyond $0.5 R_{e}$. The GaussHermite coefficient $h_{4}$ of the VP, which indicates the first order symmetric deviation from a Gaussian shape, is slightly larger than zero. Spherical as well as $f\left(E, L_{z}\right)$ axisymmetric modeling indicates that the strongly tangential motions required for a flat $\sigma_{\text {los }}$ profile in a constant $\mathrm{M} / \mathrm{L}$ model are 


\section{NGC 2434}
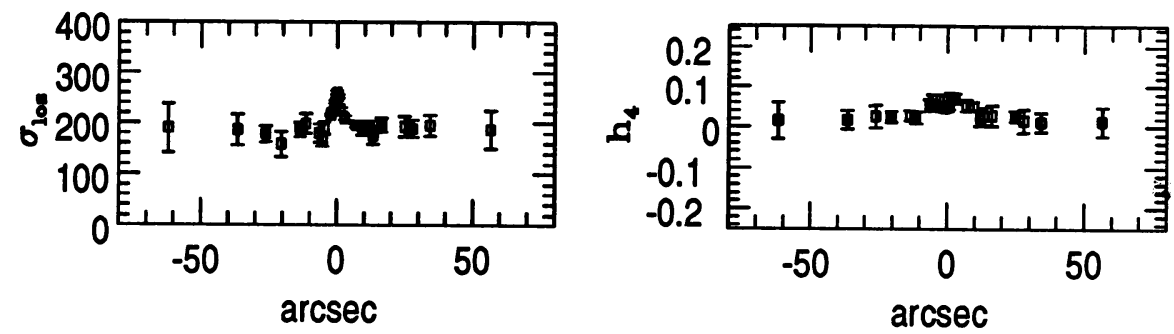

Figure 2. a) Velocity dispersion and Gauss-Hermite coefficient $h_{4}$ as a function of radius in the non-rotating E0 galaxy NGC 2434. The data extend to $2 R_{e}$. The flat $\sigma_{\text {los }}$-profile, combined with the value $h_{4} \sim 0.02$ at large radii, indicates that the first order symmetric deviation of the VPs from a Gaussian shape are small, and rule out strongly tangential anisotropy. This galaxy must therefore have a dark halo, which dominates the potential at large radii (Carollo, 1993; Carollo et al., 1994).

ruled out. NGC 2434 has a dark halo, which dominates the potential at large radii (Carollo et al., 1994).

Dynamical modeling based on the Jeans equations has received renewed attention in the context of $f\left(E, L_{z}\right)$ axisymmetric models (Binney et al., 1990). This approach uses the two-dimensional $\left\langle v_{\text {los }}\right\rangle$ and $\sigma_{\text {los }}$ fields to constrain $\mathrm{M} / \mathrm{L}$ and the inclination, while at the same time testing whether the assumed velocity anisotropy is in fact consistent with the data. Various studies provide statistical and individual evidence that $\mathrm{M} / \mathrm{L}$ increases with increasing radius (van der Marel, 1991; Carollo and Danziger, 1994a, b). This work needs to be extended to (i) check that $f$ is non-negative, e.g., by means of the methods of Dehnen and Gerhard (1994), Qian et al. (1994), or Dehnen (1994), (ii) to include the full range of velocity anisotropy, and (iii) to include triaxial models, which display a large variety in the velocity dispersion fields (Statler, 1987). Just as in the search for supermassive central black holes $(\S 1)$, it is dangerous to neglect $10-20 \%$ effects in the comparison of models with and without dark halos with kinematic data. Detailed modeling is crucial.

\subsection{INDIVIDUAL OBJECTS}

It is possible to measure radial velocities of individual globular clusters and planetary nebulae in the halos of galaxies out to distances of $15 \mathrm{Mpc}$. Studies based on globular clusters include Centaurus A, M87, NGC 4472, and NGC 1399 (Hesser et al., 1984; Huchra 1988; Mould et al., 1990; Harris, 1988; Grillmair et al., 1994). Planetary nebula measurements have been carried out for Centaurus A, NGC 4594, NGC 3115, NGC 3379, and NGC 1399 (Hui et al., 1993, 1994; Ciardullo et al., 1993; Arnaboldi et al., 1994). 
In some cases the measurements extend to about $4 R_{e}$, but the resulting velocities often do not provide very strong constraints on M/L, partly because of the modest number of measurements (Merritt and Tremblay, 1993), and partly because of our ignorance of the intrinsic velocity anisotropy ( $\$ 2.1$ ). More interestingly, the data indicate that unlike the luminous central regions the stellar halos may display substantial rotation.

Recent work has provided strong evidence for dark matter around the $\mathrm{cD}$ galaxy NGC 1399 in the Fornax cluster. The central $\sigma_{\text {los }}$ of the integrated light is $\sim 400 \mathrm{~km} / \mathrm{s}$, and translates to $\mathrm{M} / \mathrm{L}_{B}=10 \pm 3 \mathrm{M} / \mathrm{L} \odot, B$ for a distance of $15 \mathrm{Mpc}$. It falls by a factor of two at $1^{\prime}(4.5 \mathrm{kpc})$. Radial velocities of 47 globular clusters and 37 planetary nebulae show that $\sigma_{\text {los }}$ rises again to $400 \mathrm{~km} / \mathrm{s}$ at $5 ! 5(25 \mathrm{kpc})$, so that M/L ${ }_{B}=70 \pm 20 \mathrm{M} / \mathrm{L}$ $\odot, B$ inside this radius (Grillmair et al., 1994; Arnaboldi et al., 1994). This result is based on small samples, but is consistent with the recent ROSAT measurement of the X-ray emission (Jones et al., 1994), and shows that the run of mass with radius in NGC 1399 is very similar to that in M87 (Kent, 1990). Even though this establishes the presence of much dark material around NGC 1399, it is not clear whether this is the dark halo of the cD, or is the dark matter that dominates the cluster potential, or both (Grillmair et al., 1994).

\subsection{COLD GAS}

Many elliptical galaxies contain emission-line gas which extends to $\lesssim 1 R_{e}$ from the center, often appears as a warped disk, and is sometimes associated with detectable dust lanes (Shields, 1991; Buson et al., 1993). The twodimensional gas velocity field is a good diagnostic of $\mathrm{M} / \mathrm{L}$ and the shape of the inner galactic regions (e.g., Bertola et al., 1991). In some galaxies the emission-line gas rotates slower than the stars, and has a substantial velocity dispersion $\sigma_{\text {gas }}$, so that it can not be on closed orbits. A large value of $\sigma_{\text {gas }}$ in the inner few arcseconds may be caused also by seeing convolution of fast rotation, such as seen in the nuclear disk of M87 ( $§ 1)$.

There are about half a dozen elliptical galaxies with detectable HI gas which extends to many effective radii. These are ideal cases for studying the behavior of M/L at large radii (Kent, 1990; van Gorkom, 1992). Traditionally, HI velocity fields have been fitted with planar circular motion or with tilted circular ring models, just as is customary for spiral galaxies. This is incorrect for triaxial elliptical galaxies. Even though the HI extends to very large radii, deviations from circular motions caused by a triaxial halo might be significant (Lees, 1991).

Franx et al. (1994) obtained high resolution VLA data of the HI ring around the E1/S0 galaxy IC 2006, discovered by Schweizer et al. (1989). 

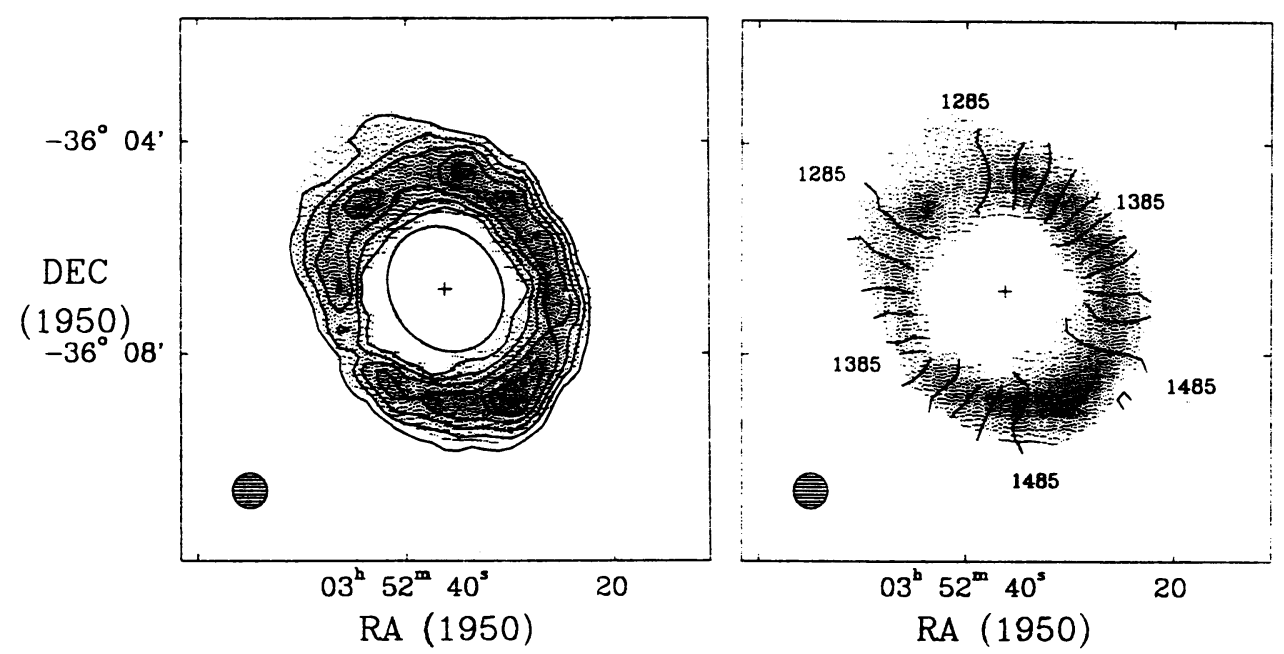

Figure 3. a) Total HI distribution in IC 2006. The contour interval is $3.4 \times 10^{19}$ atoms $\mathrm{cm}^{-2}$. The plus marks the center of the optical galaxy, and the ellipse indicates the shape of the outermost well-defined optical isophote. b) Intensity weighted mean HI velocities. The contours are spaced by $20 \mathrm{~km} / \mathrm{s}$. The systemic velocity of IC 2006 is $1385 \mathrm{~km} / \mathrm{s}$. The grey scale map is the total HI flux distribution. The filled circle indicates the beam size. The velocity variation along the ring is exactly sinusoidal, which proves the ring is a circle, seen at an inclination of $37^{\circ}$ (Franx, van Gorkom and de Zeeuw, 1994).

The remarkably regular velocity variation around the ring shows it to be essentially circular (Fig. 3). The inferred rotation curve of IC 2006 is constant between $0.5 R_{e}$ and $6.5 R_{e}(19 \mathrm{kpc})$, and $\mathrm{M} / \mathrm{L}$ increases by a factor 3.2 in this radial range. This confirms the presence of a dark halo (Schweizer et al., 1989). The settling time at $6.5 R_{e}$ is long, so if the ring is the result of infall this must have occurred in the distant past, and may be associated with the formation of the entire galaxy. If the HI has settled on a closed orbit in the equatorial plane of the galaxy, then the near circularity of the ring shows that the potential has ellipticity $\epsilon_{\text {pot }}=0.012 \pm 0.026$ in the ring plane, and that the dark mass distribution is nearly round in this plane $\left(\epsilon_{\text {dark }}=0.04 \pm 0.07\right)$. The ring kinematics do not constrain the flattening of the potential perpendicular to the ring. Models for the light distribution of IC 2006 show that it is strongly flattened in the outer parts. This suggests that it contains a nearly face-on stellar disk, and may be an S0.

\subsection{HALO SHAPES}

Simulations of the dissipationless formation of structure in the Universe generally produce triaxial dark halos (Frenk et al., 1988; White and Ostriker, 1990; Dubinski and Carlberg, 1991; Warren et al., 1992), which gener- 
ally are more prolate/triaxial than the majority of the luminous ellipticals (Franx et al., 1991). The simulations have modest radial resolution, and hence the role of minor orbit families in driving a strongly triaxial halo towards a more nearly oblate shape (Schwarzschild, 1993) may be underestimated. Moreover, the observations concern the luminous inner parts of the galaxies, but it is not clear why these should have the same shapes as the dark halos in which they are embedded.

The HI observations of IC 2006 show that this elliptical galaxy must have a dark halo that is nearly axisymmetric, and probably contains a stellar disk. This is consistent with the fact that the potentials of spiral galaxies (which are dominated by the dark matter) have small systematic elongations in the plane of the disk (Franx and de Zeeuw, 1992). The discrepancy between the shapes of observed halos and those that are formed in the simulations is most likely to be caused by the neglect of the dissipative infall of baryonic matter (Katz and Gunn, 1991; Udry, 1993; Dubinski, 1994; Navarro and White, 1994). This results in the formation of a disk, and invariably makes the halo more oblate/triaxial. It is unclear at present whether the dark halos are very flat in the direction perpendicular to the equatorial plane (Sackett and Sparke, 1990; Sackett et al., 1994), or nearly round (Steiman-Cameron et al., 1992).

\section{Conclusions}

Dynamical evidence for a central dark mass concentration requires high spatial and spectral resolution data, and detailed modeling of the velocity profiles (VPs). Ground-based absorption line measurements provide tantalizing but not iron-clad evidence for a $1.8 \times 10^{6} M_{\odot}$ dark mass in the nucleus of the non-active galaxy M32. HST $\mathrm{H} \alpha$ measurements strongly suggest the presence of a $3 \times 10^{9} M_{\odot}$ black hole in the active galaxy M87.

There is little stellar kinematic evidence for an outwards increase of $\mathrm{M} / \mathrm{L}$ inside $\sim R_{e}$ in normal elliptical galaxies. In most cases it is not possible to distinguish constant $M / L$ models with varying velocity anisotropy from models with massive dark halos. Measurement of VPs at large radii are now improving this unsatisfactory situation. Radial velocity measurements of individual objects directly probe the rotational state of the stellar halos of elliptical galaxies to radii of $\sim 4 R_{e}$, and constrain the M/L profile. Accurate mass determinations are also possible with ROSAT data.

The best kinematic tracer of the gravitational potential at large radii is cold HI gas. Analysis of the HI ring around IC 2006 shows that it has a dark halo, and provides further evidence for the continuity between disky early-type galaxies and spirals. Both appear to have oblate/triaxial halos. Dissipative infall of baryonic matter must have played an important role in 
their formation.

It is a pleasure to acknowledge stimulating conversations with Marcella Carollo, Nicolas Cretton, Marijn Franx, Roeland van der Marel and Ken Freeman.

\section{References}

Arnaboldi M., Freeman K.C., Hui X., Capacioli M. and Ford H.C., 1994, Messenger 76, 40

Ashman K.M., 1992, P. A. S. P. 104, 1109

Bahcall J.N. and Wolf R.A., 1976, Ap. J. 209, 214

Bender R., 1990, A. \& A. 229, 441

Bender R., Saglia R.P. and Gerhard O.E., 1994, M. N. R. A. S. 269, 785

Bertin G., Bertola F., Buson L.M., Danziger I.J., Dejonghe H., Sadler E.M., Saglia R.P., de Zeeuw P.T. and Zeilinger W.W., 1994, A. \& A., in press

Bertola F., Bettoni D., Danziger I.J., Sadler E.M., Sparke L.S. and de Zeeuw P.T., 1991, Ap. J. 373, 369

Binney J.J., Davies R.L. and Illingworth G.D., 1990, Ap. J. 361, 78

Buson L.M., Bertin G., Bertola F., Danziger I.J., Dejonghe H., Sadler E.M., Saglia R.P., de Zeeuw P.T. and Zeilinger W.W., 1993, A. \& A. 280, 409

Carollo C.M., 1993, PhD Thesis, Ludwig-Maxmilians Univ., Munich

Carollo C.M. and Danziger I.J., 1994a, M. N. R. A. S. 270, 523

Carollo C.M. and Danziger I.J., 1994b, M. N. R. A. S., in press

Carollo C.M., de Zeeuw P.T., Danziger I.J., van der Marel R.P. and Qian E.E., 1994, preprint

Ciardullo R., Jacoby G.H. and Dejonghe H.B., 1993, Ap. J. 414, 454

Crane P., Stiavelli M., King I.R. et al., 1993, A. J. 106, 1371

Dehnen W., 1994, M. N. R. A. S., submitted

Dehnen W. and Gerhard O.E., 1994, M. N. R. A. S. 268, 1019

Dejonghe H.B., 1987, M. N. R. A. S. 224, 13

Djorgovski S. and Davis M., 1987, Ap. J. 313, 59

Dressler A., Lynden-Bell D., Burstein D., Davies R.L., Faber S.M., Terlevich R.J. and Wegner G., 1987, Ap. J. 313, 42

Dubinski J., 1994, Ap. J. 431, 617

Dubinski J. and Carlberg R.G., 1991, Ap. J. 377, 365

Efstathiou G.P., Ellis R. and Carter D., 1982, M. N. R. A. S. 201, 975

Ferrarese L., van den Bosch F.C., Jaffe W., Ford H.C. and O'Connell R.W., 1994, A. J., in press

Franx M. and de Zeeuw P.T., 1992, Ap. J. 392, L47

Franx M., Illingworth G.D. and de Zeeuw P.T., 1991, Ap. J. 383, 112

Franx M., van Gorkom J.H. and de Zeeuw P.T., 1994, Ap. J., in press

Frenk C.S., White S.D.M., Davis M. and Efstathiou G.P., 1988, Ap. J. 327, 507.

Gerhard O.E., 1991, M. N. R. A. S. 250, 812

Gerhard O.E., 1993, M. N. R. A. S. 265, 213

Grillmair C.J., Freeman K.C., BIcknell G.V., Carter D., Couch W.J., Sommer-Larsen J. and Taylor K., 1994, Ap. J. 422, L9

Harms R.J., Ford H.C., Tsvetanov Z., Hartig G., Dressel L.J., Kriss G.A., Bohlin R., Davidsen A.F., Margon B. and Kochhar A.K., 1994, Ap. J., in press

Harris W.A., 1988, IAU Symposium 126: Globular Cluster Systems in Galaxies, eds. J.E. Grindlay and A.G. Davis-Philip, Kluwer, p.237

Hesser J.E., Harris H.C., van den Bergh S. and Harris G.L.M., 1984, Ap. J. 276, 491

Huchra J., 1988, IAU Symposium 126: Globular Cluster Systems in Galaxies, eds. J.E. Grindlay and A.G. Davis-Philip, Kluwer, p. 255. 
Hui X., Ford H.C., Ciardullo R. and Jacoby G.H., 1993, Ap. J. 414, 463

Hui X., Ford H.C., Freeman K.C. and Dopita M.A., 1994, preprint

Jones C., Stern C., Forman W., Breen J., David L., Tananbaum H., Tucker W., Franx M. and Fabian A.C., 1994, preprint

Katz N. and Gunn J.E., 1991, Ap. J. 377, 365

Kent S.M., 1990, ASP Conference Series Vol. 10: Evolution of the Universe of Galaxies, ed. R.G. Kron, Brigham Young Univ. Print Services, p. 109

Kormendy J., 1987, IAU Symposium 127: Structure and Dynamics of Elliptical Galaxies, ed. P.T. de Zeeuw, Reidel, p. 17

Kormendy J., 1993, The Nearest Active Galaxies, eds. J.E. Beckman, J. Colina and H. Netzer, Madrid, Cons. Sup. de Invet. Scient., p. 197

Kormendy J., Dressler A., Byun Y.I., Faber S.M., Grillmair C., Lauer T.R., Richstone D.O. and Tremaine, S.D., 1994, Dwarf Galaxies, ed. G. Meylan, Garching, ESO

Lauer T.R., 1985, Ap. J. 292, 104

Lauer T.R., Faber S.M., Holtzman J.A. et al., 1991, Ap. J. 369, L41

Lauer T.R., Faber S.M., Lynds C.R. et al., 1992a, A. J. 103, 703

Lauer T.R., Faber S.M., Currie D.G. et al., 1992b, A. J. 104, 552

Lees J.F., 1991, Warped Disks and Inclined Rings around Galaxies, eds. S. Casertano, P.D. Sackett and F. Briggs, Cambridge Univ. Press, p. 50

Levison H.F., 1987, Ap. J. 320, L93

Lynden-Bell D., 1969, Nature 223, 690

Merritt D.R. and Tremblay B., 1993, A. J. 106, 2229

Mould J.R., Oke J.B., de Zeeuw P.T. and Nemec J.M., 1990, A. J. 99, 1823

Navarro J. and White S.D.M., 1994, M. N.R.A.S. 267, 401

Peletier R.F., 1989, PhD Thesis, Univ. of Groningen.

Qian E.E., de Zeeuw P.T., van der Marel R.P. and Hunter C., 1994, M. N. R. A. S., submitted

Quinlan G.D., Hernquist L. and Sigurdsson S., 1994, preprint

Rees M.J., 1984, Ann. Rev. Aastron. Astroph. 22, 471

Richstone D.O., Bower G. and Dressler A., 1990, Ap. J. 353, 118

Rix H.-W., 1993, IAU Symposium 153: Galactic Bulges, eds. H. Dejonghe and H.J. Habing, Kluwer, p. 423

Sackett P. and Sparke L.S., 1990, Ap. J. 361, 408

Sackett P., Rix H.-W., Jarvis B.J. and Freeman K.C., 1994, Ap. J., in press

Saglia R.P., Bertin G., Bertola F., Danziger J., Dejonghe H., Sadler E.M., Stiavelli M., de Zeeuw P.T. and Zeilinger, W.W., 1993, Ap. J. 403, 567

Schwarzschild M., 1954, A. J. 59, 273

Schwarzschild M., 1993, Ap. J. 409, 563

Schweizer F., van Gorkom J.H. and Seitzer P., 1989, Ap. J. 338, 770

Shields J.C., 1991, A. J. 102, 1314

Statler T.S., 1987, Ap. J. 321, 113

Steiman-Cameron T.Y., Kormendy J. and Durisen R.H., 1992, A. J. 104, 1339

Stiavelli M., Møller P. and Zeilinger W.W., 1993, A. \& A. 277, 421

Tonry J.L., 1987, Ap. J. 322, 632

Trimble V., 1987, Ann. Rev. Astron. Astroph. 25, 425

Udry S., 1993, A. \& A. 268, 35

van den Bosch F.C., Ferrarese L., Jaffe W., Ford H.C. and O'Connell, R.W., 1994, A. J., in press

van der Marel R.P., 1991, M. N. R. A. S. 253, 710

van der Marel R.P., 1994, M. N. R. A. S. 270, 271

van der Marel R.P. and Franx M., 1993, Ap. J. 407, 525

van der Marel R.P., Rix H.W., Carter D., Franx M., White S.D.M. and de Zeeuw, P.T., 1994a, M. N. R. A. S. 268, 521

van der Marel R.P., Evans N.W., Rix H.W., White S.D.M. and de Zeeuw P.T., 1994b, M. N.R.A.S., in press 
van Gorkom J.H., 1992, Morphology and Physical Classification of Galaxies, eds. M. Capaccioli, G. Longo and G. Busarello. Kluwer, p. 233

Warren M.S., Quinn P.J., Salmon J.K. and Zurek W.H., 1992, Ap. J. 399, 405

White S.D.M. and Ostriker J.P., 1990, Ap. J. 349, 22

Young P.J., 1980, Ap. J. 242, 1232

GERHARD: The agreement of the predictors from the dynamical models aand the kinematical data you showed for M32 was not very impressive (equally good fits were obtained in unpublished work by Dehnen, who used different techniques but the same physical assumptions). However, what is peculiar to M32 is its high central density, so that the mass segregation time in the nucleus is very slow. Although there are no published evolution calculations for axisymmetric collisional systems, the spherical models indicate that $1.4 \mathrm{M}_{\odot}$ neutron stars, say, would have a steeper inner power law rise than the observable stars. If the extra central masswere massive remnants, one would also predict a central rise in velocity dispersion, but it would not be as steep as in tha case of a point mass

DE ZEEUW: Fully agreed.

TAYLER: Can all the theoretical work that has been done on the stochastics of stellar velocity distributions make any useful contribution to the subject?

DE ZEEUW: Yes. 